\title{
Médecins de famille et pédiatres en tête en matière de collaboration
}

\author{
Nathalie Bösch ${ }^{a}$, Esther Kraft ${ }^{a}$, Clémence Merçay ${ }^{b}$, Paul Camenzind ${ }^{b}$ \\ a $\mathrm{FMH} ;{ }^{b}$ Obsan
}

\footnotetext{
1 Etats-Unis, Allemagne, Pays-Bas, Canada, Grande-Bretagne, Australie, Norvège, NouvelleZélande, Suède. Les données de la France n'étaient pas disponibles au moment de la réalisation de ce rapport.
}

\author{
Depuis 2010, la Suisse prend part chaque année à l'enquête internationale de la fon- \\ dation américaine "Commonwealth Fund». Pour la deuxième fois, l'enquête 2015 \\ s'est concentrée sur les médecins de premier recours et a permis une comparaison \\ internationale.
}

Les soins de premier recours sont d'actualité dans de nombreux pays. De ce fait, le Commonwealth Fund [1], une fondation américaine d'utilité publique organisant chaque année depuis 1998 une enquête comparative de politique de santé, examine tous les trois ans la situation des médecins, et depuis deux éditions celle des médecins de premier recours. En 2015, l'Office fédéral de la santé publique (OFSP) et la FMH ont donc mené conjointement cette deuxième enquête auprès des médecins de premier recours établis en Suisse. Les résultats recueillis ont ensuite été évalués et analysés par l'Obsan. La plupart des questions ont été posées aux médecins de premier recours de l'ensemble des pays participants ${ }^{1}$. En collaboration avec Médecins de famille Suisse, plusieurs questions spécifiques à la situation suisse ont été ajoutées au questionnaire distribué aux médecins de premier recours suisses.

L'enquête 2015 menée par un institut externe indépendant a recueilli l'avis de 1064 des 2734 médecins de premiers recours sollicités $(69,6 \%$ de Suisse alémanique, $25,6 \%$ de Suisse romande et $4,8 \%$ du Tessin). Deux tiers d'entre eux $(68,5 \%)$ ont entre 45 et 64 ans, la part des femmes s'élève à $33 \%$, celle des hommes à $67 \%$. L'échantillon correspond à la population des médecins de premier recours en Suisse; seuls les médecins en cabinet de groupe sont surreprésentés. En Suisse, les médecins ont été relativement nombreux à prendre part à l'enquête. Aux Etats-Unis par exemple, seuls 1001 médecins y ont répondu, soit un taux de participation plutôt faible compte tenu du nombre de médecins de premier recours américains.

Les questions ont porté sur de multiples sujets ayant trait au système de santé (satisfaction, accès aux soins de santé, etc.) ou à la coordination entre les fournisseurs de soins et de prestations. Les développements suivants sont extraits de la publication des résultats détaillés de l'enquête du Commonwealth Fund
[2]. Pour l'interprétation des données internationales, il convient de tenir compte des spécificités nationales de chaque système de santé.

\section{Résultats de la Suisse en comparaison internationale}

Les résultats de la deuxième enquête internationale du Commonwealth Fund réalisée auprès des médecins de premier recours de dix pays révèlent que les médecins de famille et les pédiatres font face à une charge administrative élevée, et ils soulignent le manque de relève médicale dans notre pays. En comparaison internationale, la Suisse est championne de la coordination entre les intervenants au cours des traitements, elle occupe en effet la première place dans ce domaine; ce qui, du point de vue des médecins, a un impact positif sur les patients.

\section{Hausse générale de la satisfaction en lien avec la pratique médicale malgré la lourde charge de travail}

S'agissant de l'exercice de la médecine, le niveau de satisfaction des médecins de premier recours interrogés a légèrement augmenté par rapport à 2012 (figure 1) et le système de santé de notre pays est plutôt bien perçu. En comparaison internationale aussi, les médecins de premier recours suisses sont plutôt satisfaits de leur activité professionnelle. En 2015, ils étaient moins nombreux que trois ans auparavant à estimer que des changements majeurs étaient nécessaires pour améliorer le système de santé: ce pourcentage est passé de $49 \%$ à $44 \%$.

Malgré la diminution du nombre d'heures de travail hebdomadaires, la Suisse occupe la $2^{\mathrm{e}}$ place derrière l'Allemagne concernant la charge de travail. $60 \%$ des 


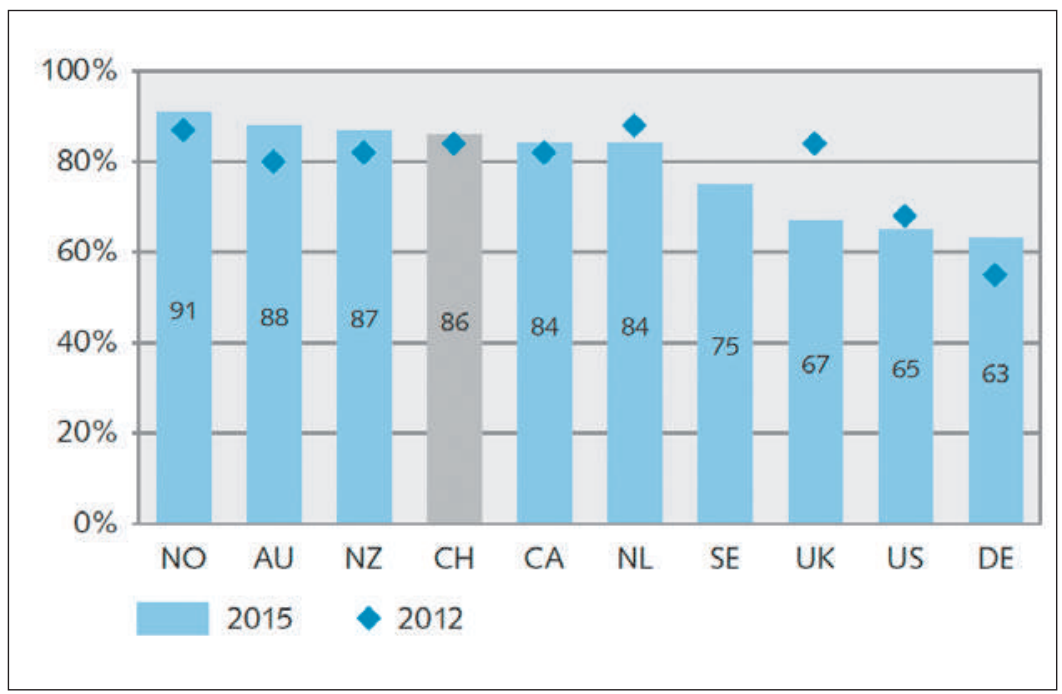

Figure 1: Satisfaction concernant l'exercice de la médecine en comparaison internationale. Source: The Commonwealth Fund, International Health Policy Survey 2015.

médecins de premier recours travaillent plus de 45 heures par semaine, contre 68\% en 2012.

\section{Résultats positifs concernant la commu- nication avec les patients, les autres spécialistes et les hôpitaux}

En comparaison internationale, les médecins de premier recours suisses se placent en tête de classement pour ce qui est de la communication avec les services d'aide et de soins à domicile. Pas moins de $74 \%$ des médecins de premier recours suisses sont très satisfaits de la coordination avec ces services (ils la considèrent comme «facile» ou «très facile»), tandis que la moyenne pour les autres pays se situe autour de $38 \%$.

La coordination entre les médecins de premier recours et les autres spécialistes fonctionne également très

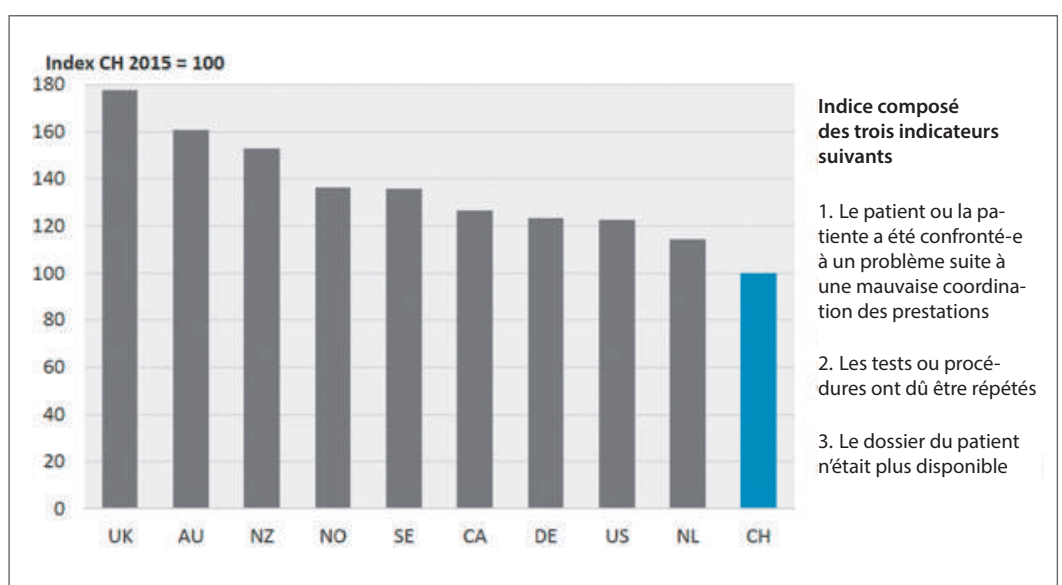

Figure 2: Indice des problèmes survenus suite à une mauvaise coordination en comparaison internationale.

Source: The Commonwealth Fund, International Health Policy Survey 2015. bien: la quasi-totalité des médecins de premier recours (94\%) indiquent qu'ils reçoivent "toujours" ou "souvent» un rapport du spécialiste avec toutes les informations pertinentes concernant leurs patients. S'agissant de la coordination avec les hôpitaux, la Suisse occupe également une très bonne place: $82 \%$ des médecins de premier recours reçoivent «toujours» ou "souvent» des informations concernant l'hospitalisation de leurs patients. De l'avis des médecins, ces bons résultats se reflètent également au niveau de la qualité des soins car selon eux, il est rare que des patients suisses aient des problèmes en raison d'un manque de coordination (figure 2).

Par rapport aux médecins des autres pays, les médecins suisses estiment moins fréquemment que les patients sont soumis à des délais d'attente. Sur ce point, notre pays occupe encore en 2015 la tête du classement. La disponibilité des médecins de premier recours est bonne, malgré une tendance à la baisse depuis 2012. En 2015, 78\% (2012: 86\%) des médecins de premier recours ont pu accepter de nouveaux patients et 54\% (2012: $62 \%)$ ont été en mesure de recevoir des patients qui demandaient un rendez-vous dans un délai court.

En comparaison internationale, les médecins de premier recours suisses sont ceux qui déclarent le moins souvent que leurs patients ont été confrontés à des problèmes liés à un manque de coordination entre les divers fournisseurs de soins ou que des examens ont été refaits inutilement en raison de l'indisponibilité des résultats de tests précédents.

Les médecins de premier recours se montrent globalement plutôt sceptiques vis-à-vis des nouveaux instruments actuellement débattus (par ex. Disease Management, Case Management) et visant une meilleure coordination. En 2015, 65\% d'entre eux ont émis sur ce point au moins trois réserves. Ce scepticisme à l'encontre des nouveaux outils peut certainement s'expliquer par la grande satisfaction des médecins de premier recours concernant leur situation actuelle et leur bonne position en comparaison internationale. Selon eux, le principal problème réside dans le niveau élevé des coûts.

\section{Moins de temps pour le patient à cause de la charge administrative}

Un médecin de premier recours sur deux estime que les tâches administratives liées aux assurances représentent un problème majeur; un sur trois considère comme problématique le temps consacré à la saisie des données cliniques ou à la collecte de données de qualité pour les institutions étatiques ou autres. Par rapport à l'enquête de 2012, la part des médecins de 
premier recours en mesure de consacrer au moins $75 \%$ de leur temps de travail aux patients a nettement diminué: cette part est passée de $60 \%$ en 2012 à seulement $45 \%$ en 2015 . Cette tendance correspond également aux résultats d'autres études qui révèlent une augmentation de la charge administrative.

\section{Potentiel d'amélioration dans l'utilisa- tion des instruments de cybersanté}

Malgré une progression marquée du dossier médical informatisé ces trois dernières années chez les médecins de premier recours suisses (de $41 \%$ à $54 \%$ des médecins), notre pays se maintient en fin du classement international dans ce domaine avec une évolution très hétérogène. On relève une utilisation plus fréquente du dossier médical informatisé chez les médecins de premier recours de moins de 45 ans ainsi que chez les médecins travaillant en cabinet de groupe. Il existe également des différences régionales. C'est en Suisse alémanique que le dossier médical informatisé est le plus répandu et au Tessin qu'il est le moins utilisé.

D'autre part, tous les instruments de cybersanté, dont ne fait pas partie le dossier médical informatisé, ne connaissent pas le même degré de diffusion. Ainsi, $80 \%$ des médecins de premier recours suisses offrent à leurs patients la possibilité de prendre contact avec eux par courriel. On constate également des améliorations dans la communication électronique avec les laboratoires et les autres spécialistes. Par contre, les médecins de premier recours ne sont que $24 \%$ à être en mesure d'effectuer des transferts électroniques d'ordonnances à une pharmacie. Dans $41 \%$ des cas, le fax reste le moyen par lequel les médecins de premier recours reçoivent des informations des hôpitaux. Seuls $19 \%$ d'entre eux reçoivent ces informations par courrier électronique.

\section{Manque de relève général}

La Suisse est touchée par un manque important de jeunes médecins de premier recours alors que près d'un tiers des médecins de famille est âgé de 60 ans et plus. Parmi eux, seuls $20 \%$ disposent déjà d'un successeur pour leur cabinet. Cette pénurie a deux facettes. D'une part, elle s'explique par le nombre insuffisant de places d'études proposées en médecine humaine depuis une vingtaine d'années. Les facultés de médecine ont depuis reconnu la nécessité d'agir et le nombre de places d'étude est en augmentation depuis 2008. Mais il faudra attendre une douzaine d'années avant que les étudiants qui entament actuellement leur formation puissent pratiquer à titre indépendant. La Suisse devra donc relever d'importants défis ces prochaines années pour couvrir les besoins de la population en soins de premier recours.

La seconde facette concerne l'attractivité de la profession de médecin de premier recours. Leur revenu comparé à celui des autres spécialistes ainsi que la lourde charge administrative sont des facteurs susceptibles d'avoir une influence négative sur l'attractivité de la profession. Pourtant, les médecins de premier recours suisses sont aujourd'hui plus satisfaits de leur revenu qu'il y a trois ans (72\% en 2015 contre 57\% en 2012). Mais l'enquête de 2015, comme celle de 2012 déjà, révèle qu'une insatisfaction prévaut lorsque le revenu est comparé à celui des autres médecins spécialistes: seuls 19\% des médecins interrogés s'estiment satisfaits.

La relève de médecins de premier recours: le grand défi des années à venir.

\section{Remarque finale}

Les médecins de premier recours suisses sont satisfaits de leur activité médicale et, de manière générale, ils sont bien placés en comparaison internationale. Cette satisfaction a même augmenté par rapport à 2012. Ils sont en tête de classement concernant la communication et la coordination avec les spécialistes et les organisations d'aide et de soins à domicile. Le résultat est également excellent concernant les courts délais d'attente et la disponibilité des médecins de premier recours.

Un point dont les médecins de premier recours suisses ne sont pas du tout satisfaits concerne leur revenu en comparaison avec celui des autres spécialistes. La lourde charge administrative représente également un problème majeur. Enfin, le temps de travail hebdomadaire en Suisse est très long en comparaison internationale, malgré une tendance à la baisse. Le recours aux outils de gestion du cabinet médical (indicateurs et comparaisons) ainsi que l'utilisation et la diffusion des instruments de cybersanté présentent un potentiel d'amélioration. Le principal enjeu des prochaines années demeure cependant la pénurie de médecins.

Ces analyses sont extraites de la publication des résultats détaillés de l'enquête internationale sur la politique de la santé de 2015 du Commonwealth Fund [2]. La FMH se tient à votre disposition pour tout complément d'information (ddq[at]fmh.ch ou 03135911 11).

\footnotetext{
Références

1 www.commonwealthfund.org

2 http://www.bag.admin.ch/themen/gesundheitspolitik/index. html?lang=fr
} 